\title{
Health Policies Require New Multidisciplinary Research
}

\author{
Pedro Guedes de Carvalho
}

\section{Introduction}

The world trends in human life activity highlight that health is beyond a doctor, a hospital or an illness problem. Feeling healthy really impacts productivity and is also very important to fully understand human wellbeing, physical and mental personality, and consequently policies. In health policy the "diagnosis" is not always suitable and this will sometimes open incentives for wrong policy responses.

According to Silva (2012), ${ }^{1}$ we should consider three important features relating to health policies in Portugal: sustainability and financial efficiency; equity in access and results and system quality. Taken as a whole, all the three features are connected with "cost" management. In fact, since the 1970's in $20^{\text {th }}$ Century Portugal, the National Health System was implemented as a democratic achievement guaranteeing free health access to every citizen, independent of race, socioeconomic or religious status. It was a democratic political decision after the revolutionary process within a context of economic expansion free of special financial constraints. Suddenly the health care system changed rapidly: higher salaries, a huge increase of new infrastructures and demand for health services and an exponential use of new and expensive technologies. If we link this to the new international and multinational sector of pharmacy and the changes in demographic trends, namely higher life expectancy rates and ageing society, we have the right environment where designing policies would include extremely delicate financial strategies.

Furthermore, new physical and nutritional habits were induced through new technology based industries after World War II. The last 70 years brought

1 Silva, M. V. (Maio de 2012). Políticas Públicas de Saúde: Tendências recentes. Sociologia, Problemas e Práticas no.69 
important changes in ways of life, physical activity and its interaction with health services.

In rural societies, human activity was physically demanding. Long distance commuting between home and workplace consumed people's time and energy, leaving little room for attention to their health. Nowadays, work usually requires minimal levels of physical energy but good competence and psychological efforts that may lead to difficulties to concentrate and stress, which may in its turn lead to psycho-somatic dysfunctions. In addition, with lesser time for sport activities, workers do not exercise enough to restore their feelings of complete health - which is different to being ill. In sum, health is not simply the absence of illnesses in society; we are defining new meanings of 'health', 'wellbeing' and 'happiness', which requires a broad range of approaches and different instruments to lessen the costs of regular and traditional national health systems.

Today, in a large number of societies, information comes faster than our brains' capacity to digest it. Societies are facing ageing processes ${ }^{2}$ and chronicle diseases (hypertension, diabetes, arthritis and posture) with obesity prevalence. The interaction between school and families has changed dramatically and while more children can access education, families have not adapted accordingly to support healthier daily habits. Young people live alone, isolated, when making their consumption choices, and over $64 \%$ of the Portuguese population do not engage in physical exercise. As a consequence, usually avoidable diseases will emerge, making a demand on the National Health System. Financially, when people live longer the demands increase on the National Pensions System. High tech innovations will diminish the need for workers with demands on the National Labour/Security System. Generally, public policies need to be innovative to meet this challenge.

\section{Proposal}

The aim of this article is to underline the need for researchers from different disciplines to work together. The main health issues for a country or a National Health System have been too much focused on illnesses instead of health, wellbeing and happiness, mono-disciplinary dealing with the costs of illness or producing statistics on hospital expenditures and medical prescriptions. However, Health policies are not just a matter for doctors, hospitals and pharmacies. They should also be designed to deal with a more human, holistic understanding when people who ask for help experiencing illness or pain and

2 David A, W. (2007). Economics of Ageing Program. NBER, Report 4. 
complimentary approaches would be helpful. The theories, questions and methods of a number of disciplines are relevant for the studying and design of health systems. Too many health problems do not find efficient solutions when they are too much restricted to what hospitals and other health services can provide. We need a wider approach to find new, efficient financial solutions for sustainable solutions of the population's need for health. ${ }^{3}$

\section{Examples of the multidisciplinary rationale}

Economists use to look and believe in facts; facts are our basic data. Usually data is expressed through numbers. However, besides data we have to be open to other important issues such as the way data was collected and extracted, the reason why we use additional information to improve the quality of our analysis.

Let us take a simple example of what could happen to one Portuguese citizen that feels sick or experience a painful situation thinking he needs health care. This simple situation gives rise to a number of decisions by the health consumer, each one with slightly different impact on costs for the public health and care systems. If we decompose this possible process we will be able to define adequate strategies.

First of all the sociological viewpoint oblige us to consider the social status of that citizen and the system to which he is part of. Secondly, someone has to define the exact physical and psychological status, i.e. the visible and invisible pain and emotional status. In a way, to find out how severe the complaints are in order to filter them down and establish the adequate path for observations. Thirdly, the time of assistance comes, usually spent with a doctor. At this point the effective relationship between citizen and health system will start. The doctor is the only authority that can define what is going to happen afterwards - which is too much responsibility for emerging excessive or negligible costs to a single person. The system itself defends their staffs with regular protocols for each described illness or complaint, which looks understandable. Fourthly, we have the technological question. In order to be completely safe defence against hard legislation, doctors do prefer to prescribe additional examinations before they give the illness verdict (double competencies). In the past, very well prepared doctors had to define diseases with simple and more natural clinical instruments. Simple body touching, contextual information and storytelling would be enough to accurate diagnosis. Technological development has led to

3 WHO. (2003). Making Choices in Health:WHO Guide to cost-effectiveness analysis. T. Tan-Torres Edejer, R. Baltussen, T. Adam, R. Hutubessy, A. Acharya, D.B. Evans and C.J.L. Murray (eds). Geneve: WHO 
rising costs and given birth to a rapidly growing market for technological and informatics firms, which produce new machinery offered to hospitals and promising to enhance the quality of diagnosis. And this becomes a cumulative process, when technology reduces doctors' own judgments, making them more dependent on laboratory instruments and permanent updating of skills. In the past we had more simple and costless diagnosis for most of the hospital entrances. Today we replaced it by a technological, costly system of diagnosis. Naturally, we do not defend conventional medical practices just because they are not compatible with new technologies, the latter are needed and represent indispensable scientific achievements. The remedy is to re-train health professionals in lean diagnosis methods and a deeper understanding of patients' emotions and needs.

Most decisions of these professionals also depend on the consumers' type of health care system, being either private or public, designed for the rich or for the poor. Access to health provision should be for everyone. If not well designed, these financing medical costs would be overpaid later. Consultations, examinations and other medical interventions could be paid differently, depending on the social and health needs of the consumers. This is why a new multidisciplinary approach should be applied to study and propose new health policies and measures.

\section{Questions in the Agenda}

A number of important questions can, in this context, be raised:

- How do social demographic changes transform economic markets?

- How do cultural and regional aspects do affect social behavior, and how should that have an influence upon decisions concerning the demand for health services?

- How can different types for the provision of health service alter future demand for the health service system?

- What do we know about the provision of new, alternative (or complimentary) health services?

- When might physical exercise be an effective prescription for better health?

- How do pharmaceutical and chemical laboratories operate in the economy? 
- How does this multinational, industrial complex function and affect health professionals' decisions?

- Who should pay for the health care systems?

Many other markets - e.g. education and training, recreation and leisure, housing, transportation, food, pharmaceutics, etc. - must be developed and adapted to the process of demographic ageing. These transformations will also influence the whole macro economy, its GDP, productivity, per capita income, consumption patterns and socioeconomic growth.

New research projects should also attempt to integrate into their measurements of health these multiple domains, such as physical and psychological functioning, mental health, access, equity and the particular conditions that encompass overall health like wellbeing and happiness. The project producing the articles in this special issue have already taken a first step towards such an integrated effort. It has followed in two tracks: one is focused on aggregate measurements, another on condition-specific analysis.

Figure 1. Agents and health care actions

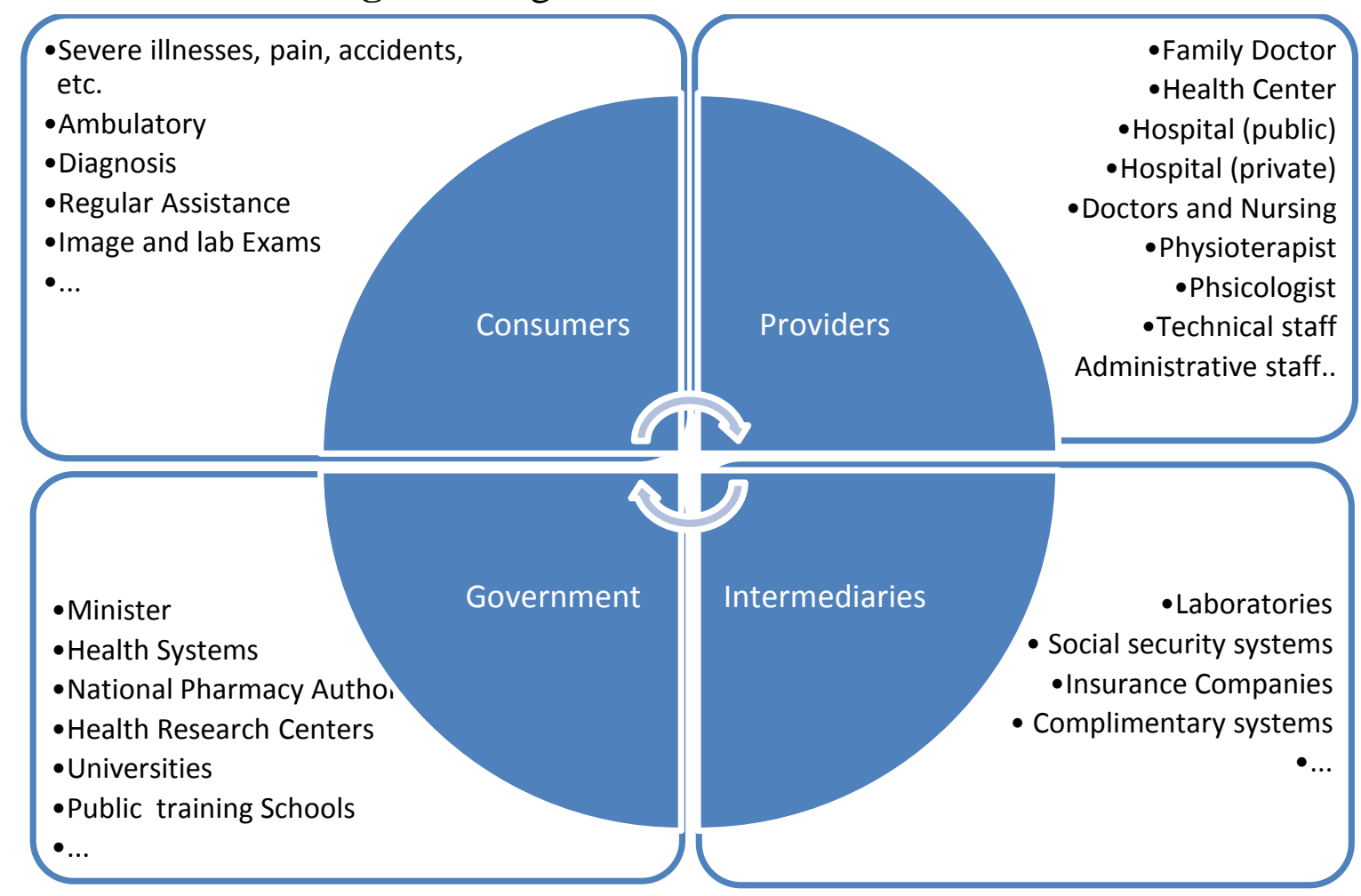

Source: Health policies require new multidisciplinary research: By Pedro Guedes de Carvalho.

Our hope is that the collaborative effort will be further enhanced by an outreach component, engaging academics, health care professionals, consumers of health care systems and government officials, by the establishment of practically useful 
national health accounts. Otherwise, national health systems financing would be less sustainable.

\section{Proposal}

Since cost efficiency is a major issue when promoting the sustainability of health systems, every research field should be involved in the design of a new health policy. Every discipline should provide its specific approaches, opening a wide discussion in order to find network instruments able to impact cost structure.

A multidisciplinary approach fits the study of real, complex situations, indeed a characteristic of a task to make health provision sustainable, equally accessible and with good quality.

Figure 1 gives an image of the agents involved in the health system. These actors are not homogeneous, must sometimes be decomposed and studied apart, can represent different interests and decision-behaviour to be considered in policy making. A seemingly innocent, even positive situation or intervention in one of the fields might be hampered or completely void by conditions or events in one of the other fields.

For the sake of simplicity we do not consider the completely market oriented system, where each citizen consults a doctor in the private sector for the first visit and diagnosis, whilst the overall system shares the expenses according to each individual's own health care arrangement and socioeconomic condition.

Figure 1 is mostly applicable to national health conditions in Portugal. Other or new agencies can be included, as well as different conceptions, disciplines, issues or problematic topics. Here, we focus on people or activities that are supposed to carry the major part of the health costs.

In the figure, the four quadrants represent examples of types of agents and types of actions. These refer to a functioning market with consumers and providers of public services, i.e. the regular type of interventions either severe, accidental, regular or consequences of past interventions.

In order to further enhance research on the foundations for health policies, we must pay more attention to existing possibilities within the systems and actions of the consumer. Theoretically, the set of those opportunities are described within each quadrant. In practice, all of them can occur without special enforcements. As a consequence, several financial costs are differently associated with each decision.

We might raise the question of family doctors: Is it a question of equity of access or a financial? When does the system save more resources? If we go straight to the hospital, will they assist us? Are we doubling the expenses? 
The second, lower, set of quadrants refer to the governance system and the intermediaries interconnecting perfectly. Usually, health policy is seen as the business of only one ministry, i.e. the minister is responsible for the whole health administration from infrastructure to staff labour conditions and system regulation. But social security systems are also highly connected and should be studied and approached together. If we want to pursue the equity in health accessibility, different solutions may be necessary for apparently the same problems, depending on demographic trends, regional situations, composition of the labour force or age-structure.

Between actions in the lower quadrants, a very important game takes place, involving private interests of provider firms and public interest of good quality service and cost-efficiency. This should be given much attention, since private agents do intervene in the provision of machinery, medical instruments, equipment, pharmaceutical products and services, established protocols and other items. How are, for instance, public competitions regulated? Or who decides if a new drug is better than one used up until today? Is there a policy of cheaper medicine for the poor (generics) than for the rich? Is actually health uncomplicated merchandise that should be traded and regulated exclusively by prices and the market? Is public intervention necessary to guarantee equal access for all? As we can see, several issues must be addressed before we settle for the most adequate health policies.

Another concern exposed by a multidisciplinary approach is that practically every implemented policy will influence the agents' behaviours. They will try to counteract and offset potentially negative effects, which - if wanted and needed - requires reinforcement procedures. In the ideal multidisciplinary setting, every question should be put to participants representing all disciplinary angles, gathering, integrating and synthesizing their feedback.

In Figure 1, all research approaches have to be tested on each of the segments, disclosing the interconnection between such fields as medicine, technology, economy, sociology, psychology, physiology, market, prices and costs, etc. As well as today's new trends of retirement, new labour market constraints, new and more genetically complex diagnosis for exquisite illnesses, and several other developments must be part of the contextual understanding. ${ }^{4}$

\section{Conclusions}

Most universal health policies are being centred on cost efficiency approaches, in addition to a more recent concern with equity and sustainability of the

4 David A 2007 
systems. However, too few improvements are noticed and countries are simply cutting public spending without qualified information. It means high risks of under-performance.

The main reason for these conditions is that the governance is mostly focused on illnesses, pain, surgeries, medicine and chemical treatments. We argue for an approach with more focus on the human being, wellbeing and happiness. Sometimes people do not need to visit a hospital or a doctor's consultation but can be treated in a more efficient and cost-effective way. We presented a 'industrial diagram' interpreting health related actions, proposing an interdisciplinary approach, trying to find where the cost is and suggesting more socially efficient and qualified network solutions, where every disciplinary voice is listened to.

Pedro Guedes de Carvalho has a PhD in Economics and is the actual Dean of a Human and Social Sciences Faculty at University of Beira Interior - Portugal 


\section{References}

David A, W. (2007). Economics of Ageing Program. NBER, Report 4.

Silva, M. V. (Maio de 2012). Políticas Públicas de Saúde: Tendências recentes.

Sociologia, Problemas e Práticas no.69.

WHO. (2003). Making Choices in Health:WHO Guide to cost-effectiveness analysis. T. Tan-Torres Edejer, R. Baltussen, T. Adam, R. Hutubessy, A. Acharya, D.B. Evans and C.J.L. Murray (eds). Geneve: WHO. 